\title{
Artificial neural network model and fuzzy logic control of dissolved oxygen in a bioreactor
}

\author{
Nor Hana Mamat ${ }^{1}$, Samsul Bahari Mohd Noor ${ }^{2}$, Laxshan A/L Ramar ${ }^{3}$, Azura Che Soh $^{4}$, \\ Farah Saleena Taip ${ }^{5}$, Ahmad Hazri Ab. Rashid ${ }^{6}$ \\ 1,2,3,4 Department of Electrical \& Electronic Engineering, Faculty of Engineering, Universiti Putra Malaysia, Malaysia \\ ${ }^{5}$ Department of Food and Process Engineering, Faculty of Engineering, Universiti Putra Malaysia, Maaysia \\ ${ }^{1}$ Department of Automation, Faculty of Electrical and Automation Engineering Technology, \\ TATI University College, Malaysia \\ ${ }^{6}$ Industrial Biotechnology Research Centre, SIRIM Berhad, Persiaran Dato Menteri, Malaysia
}

\begin{tabular}{l} 
Article Info \\
\hline Article history: \\
Received May 20, 2019 \\
Revised Aug 21, 2019 \\
Accepted Sep 5, 2019 \\
\hline Keywords: \\
Artificial neural network \\
bioreactor \\
Dissolved oxygen \\
Fuzzy logic control \\
Penicillin
\end{tabular}

\begin{abstract}
In a fermentation process, dissolved oxygen is the one of the key process variables that needs to be controlled because of the effect they have on the product quality. In a penicillin production, dissolved oxygen concentration influenced biomass concentration. In this paper, multilayer perceptron neural network (MLP) and Radial Basis Function (RBF) neural network is used in modeling penicillin fermentation process. Process data from an industrial scale fed-batch bioreactor is used in developing the models with dissolved oxygen and penicillin concentration as the outputs. RBF neural network model gives better accuracy than MLP neural network. The model is further used in fuzzy logic controller design to simulate control of dissolved oxygen by manipulation of aeration rate. Simulation result shows that the fuzzy logic controller can control the dissolved oxygen based on the given profile.
\end{abstract}

Copyright $@ 2020$ Institute of Advanced Engineering and Science. All rights reserved.

\section{Corresponding Author:}

Nor Hana Mamat,

Department of Electrical \& Electronic Engineering,

Faculty of Engineering, Universiti Putra Malaysia,

43400 UPM Serdang, Selangor Darul Ehsan, Malaysia.

Email: norhana@tatiuc.edu.my

\section{INTRODUCTION}

Bioreactor is known as vessel or tank in which a chemical process is carried out that involves organisms or biochemically active substances derived from such organisms. Bioreactor provides a controlled and suitable environment for microorganism to grow, reproduce and synthesis product. It is used to manufacture a wide range of useful biological products via fermentation process, among others ethanol [1], antibiotics [2], protein production [3], vaccines [4] and bioplastic [5]. In bioprocess, biological components are sensitive to environmental changes in the bioreactor. Variations in $\mathrm{pH}$, temperature, dissolved oxygen (DO) and nutrients may adversely affect process reproducibility or cell activity [6].

Dissolved oxygen is a key macro-nutrient for the microorganism growth, metabolic production and maintenance [7]. Functional relationship between the process variables in a penicillin fermentation introduced in [8] states that dissolved oxygen, $\mathrm{pH}$ and temperature influenced biomass concentration, while dissolved oxygen concentration is related to $\mathrm{pH}$ and temperature. Findings from experimental study suggests biomass growth is highly dependent on both glucose as the carbon source and oxygen as substrates [7]. Control of environmental variables (temperature, $\mathrm{pH}$ and dissolved oxygen) is important in a fermentation process. Proteins' reaction is slow if temperature decreases and if it rises the characteristic properties of the protein will be altered. A suitable $\mathrm{pH}$ level is needed for transfer of feedstuff to cell membrane and energy out of the cell [9]. 
Commercial production of antibiotics such as penicillin is through fermentation in fed-batch bioreactors. In fed-batch fermentation, substrate is added into the bioreactor during the batch run while cells and products are only withdrew at the end of fermentation run [10]. It is a preferred mode of fermentation as it combines the advantages of batch and continuous modes. Compared to batch mode, improved overall productivity can be achieved using fed-batch as it offers convenience in terms of better control over variation in substrate concentration and differentiation of growth [11].

Control of fed-batch bioprocess is challenging as accurate mathematical model is unavailable, also random variations occur in initial process state and model parameters from batch to batch. Lack of understanding on the microorganism's complex regulatory network and the interactions in bioreactor contributes to the absence of reliable mechanistic model [12]. An alternative to mechanistic model of penicillin fermentation is data- driven models using historical data of the process. Unlike mechanistic models, knowledge of detailed process description is not necessary as it only depends on the availability of a large process database [10]. Collection of large amounts of data is possible with the use of modern instrumentation techniques in industrial scale fed-batch bioreactors.

Artificial neural network is a data-driven method that has been successfully applied in predicting system behavior based on measured variables in a fermentation process because it can arbitrarily approximate the nonlinear behavior of the process [13]. Previous studies on the use of ANN in fermentation include rumen fermentation [14], acid synthesis [15], xanthan gum [16], foreign protein [17], E-coli fermentation [18], yeast fermentation [19] and solid-state fermentation [20]. Multilayer perceptron neural network (MLP NN) and radial basis function neural network (RBF $\mathrm{NN}$ ) are two of the most frequently used neural network structure, and thus chosen for model development in this work. Fuzzy control is designed to deal with uncertainties in a system as normally arises in a fed-batch fermentation due to nonlinear behavior of the process. Unlike model-based method, fuzzy logic control does not utilize complex model and initial knowledge on the dynamics of the system is not required [21].

In this paper, Multilayer Perceptron (MLP) and Radial Basis Function (RBF) neural network models are developed using real process data from an industrial fed-batch penicillin fermentation. The neural network model is then used in place of the process model in a fuzzy logic control of dissolved oxygen by manipulating aeration rate to the fed-batch bioreactor

\section{RESEARCH METHOD}

The process of modelling the bioreactor and involves a few important steps as outlined in the flowchart of Figure 1 and Figure 3. Variable selection is done before building neural network models for further use in fuzzy logic control of dissolved oxygen. This is to ensure only input variable that has influence on the output variable is used in modelling the process. Two Artificial Neural Network been implemented for modelling, namely the Multilayer Perceptron (MLP) and Radial Basis Function (RBF). Fuzzy logic controller design involves fuzzification process and defuzzification process.

\subsection{Variable Selection}

Process data used in this paper is obtained from a study on development of realistic simulator for process control and optimization study of an industrial penicillin fermentation process [22]. The fed-batch bioreactor is $100,000 \mathrm{~L}$ in volume and fitted with sensors for online measurements of $\mathrm{pH}$, dissolved oxygen (DO), temperature, pressure and foaming. A total of 10 fermentation batches is available. 4 batches will be used for neural network training, one batch for neural network testing and another batch is used as reference profile for fuzzy logic controller.

The first step in modelling is to determine which input variable will affect the DO level of the bioreactor and penicillin concentration. Previous studies state that input variables affecting the DO level are aeration rate $(\mathrm{L} / \mathrm{m})$, pressure (bar), water injection rate $(\mathrm{L} / \mathrm{m})$, substrate feeding rate $(\mathrm{L} / \mathrm{m})$ and temperature (Celsius) [22-23]. Sensitivity analysis is performed on the process batch data using cosine amplitude method (CAM) to determine the relative effect of each process variable towards the output variables; dissolved oxygen and penicillin concentration, as defined by the value of Rij in (1) [24]. A larger value of Rij indicates a stronger influence of the input variable xi towards an output variable yj in m-dimensional space data pairs [25].

$$
R_{i j}=\frac{\sum_{k=1}^{m} x_{i k} y_{j k}}{\sqrt{\sum_{k=1}^{m} x_{i k}^{2} \sum_{k=1}^{m} y_{j k}^{2}}}
$$




\subsection{Multilayer Perceptron ANN}

Multilayer Perceptron neural network consists of a series on interconnected nodes or neuron as shown in Figure 2. These neurons are essentially nonlinear processing elements which represent a nonlinear transformation on the summation of the input signals [5]. The nodes are connected by a set of scalar weights. There is always an input and an output layer to the network and the number of neurons in both these layers depends on the respective number of inputs and outputs being considered. In contrast, the number of hidden layer neurons may vary from zero to any finite number. The number of neurons in each hidden layer is also user-specified [6].

The MLP network is coded in MATLAB software. The model trained using data sets previously acquired from real bioreactor. Two data sets are created from the batch data, called training data and testing data. The training data will be used to train the neural network repeatedly until it reaches acceptable error range. Meanwhile testing data will be used to verify trained neural network. The training data and testing data should not contain same data sets. Advisably, $80 \%$ of available data used for training and remaining $20 \%$ used for testing. In this case, data from batch 2, batch 3, batch 9 and batch 10 used for training and meanwhile, data from batch 8 used for testing. Another matter that should considered is the structure of MLP ANN, specifically the number of hidden layers and neurons for each layer. There is no specific method to determine the optimum number of hidden layers and neurons of each layer. Thus, the training process is repeated many times with different combination of number of hidden layers and neurons of each layer. Best structure is chosen based on the performance of each MPL structures, which was [15, 30] as shown in Figure 3. Flowchart for RBF neural network as shown in Figure 4.

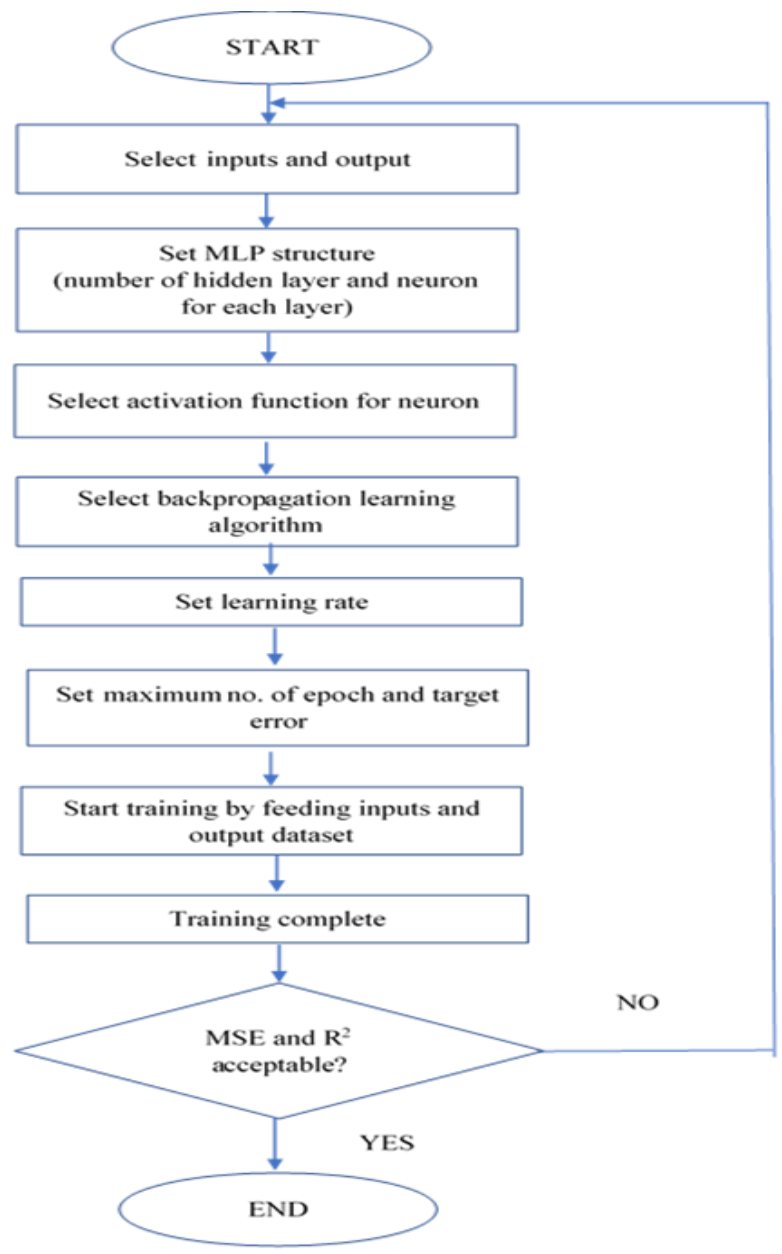

Figure 1. Flowchart for MLP neural network

There are few parameters that should be set for the training. The learning process continues as the data sets are presented repeatedly to the ANN and weights between the nodes are adjusted to reduce the error. 
Epoch is the number of times the same data set being fed into the ANN. While training the ANN, the maximum number of epochs should be set, so that the training will stop after a certain number of epochs. Failure to set this training parameter may result in non-stop training process. In this study, we set the maximum number of epochs to be 1000 . Another parameter that should be set is the error goal. The training process continues until the maximum number of epochs is reached or the error met the error goal, whichever comes first. Learning rate is another parameter which controls the speed of the learning of the ANN. In back propagation, the learning rate is analogous to the step-size parameter from the gradient-descent algorithm. If the step-size is too high, the system will either oscillate about the true solution, or it will not diverge completely. If the step-size is too low, the system will take a long time to converge on the final solution. The learning rate is set to 0.1 .

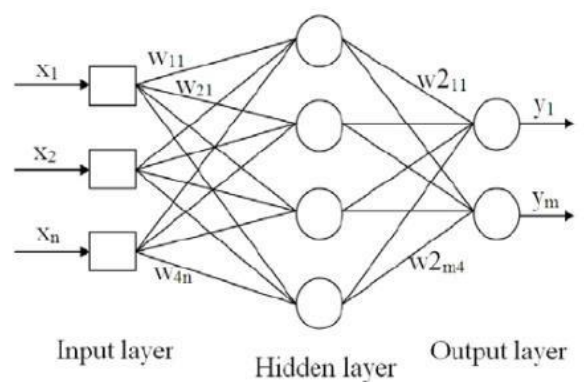

Figure 2. Multilayer Perceptron Artificial Neural Network

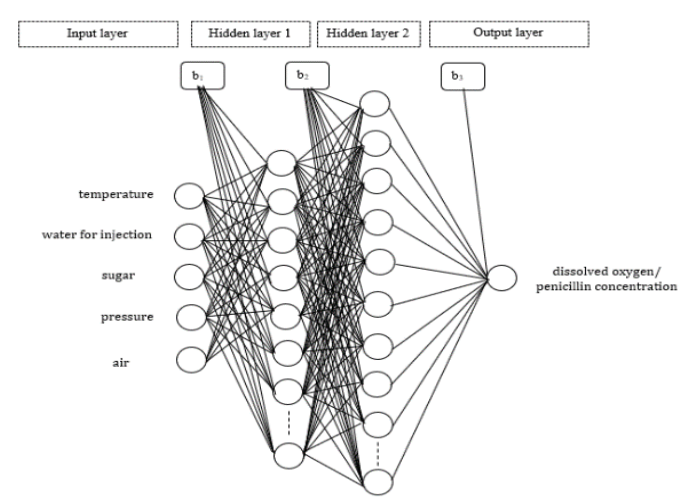

Figure 3. MLP neural network structure

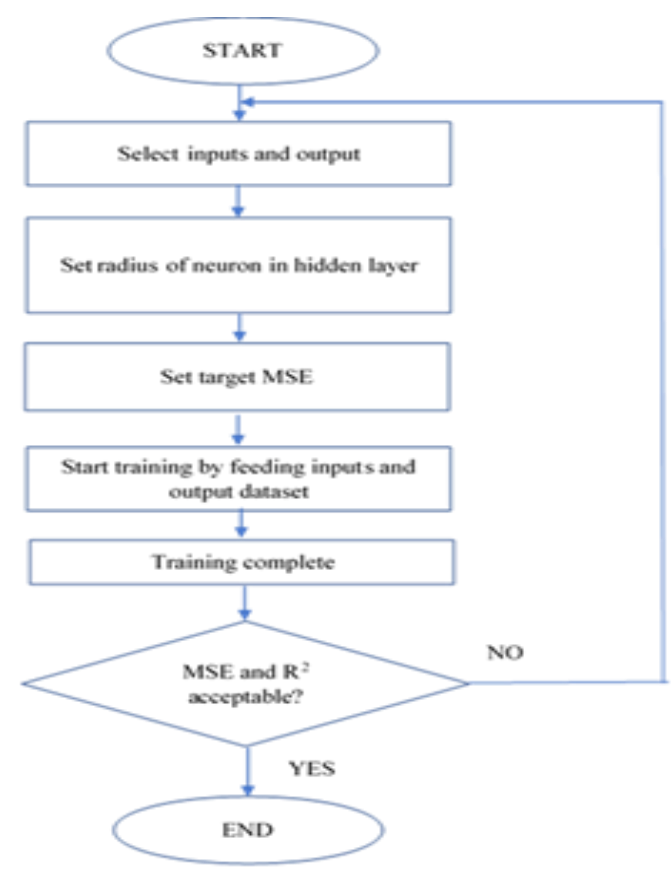

Figure 4. Flowchart for RBF neural network

\subsection{Radial Basis Function ANN}

$\mathrm{RBF} A N N$ is a forward neural network which uses radial basis functions, $\Phi \mathrm{m}($.$) as activation$ functions for each hidden layer neuron and the output of RBF ANN is weighted linear superposition of these basis functions [7]. Figure 5 shows the structure of RBF ANN. 
The process of selecting data for both training and testing tests are the same with the MLP ANN process. Nevertheless, the structure of RBF has different approach compare to MLP. Unlike MLP, it has only one single layer which consists of numbers of neurons. The parameter to be adjusted is the spread of each neural. Similar like MLP, there is no method to find optimum spread that will give best performance. Thus, the training process will be repeated with different radius value and compared to choose the one with more accurate result.

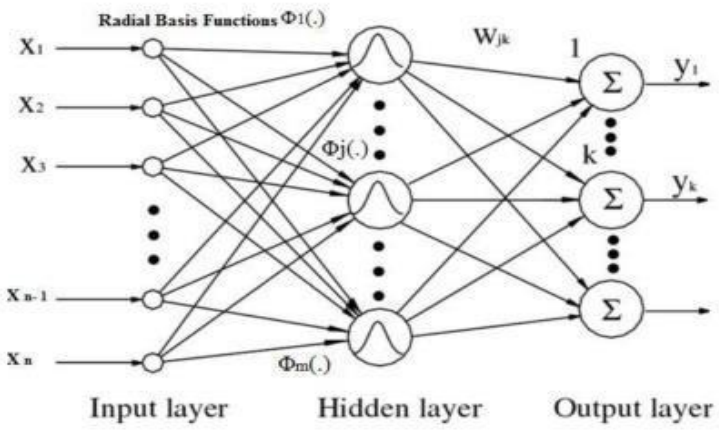

Figure 5. The basic structure of RBF neural network

\subsection{Designing the Valve}

The aeration level can be controlled using limited integrator in the simulated valve subsystem as shown in Figure 6. Note that the valve is directly controlled by the fuzzy logic controller. The DO level shouldn't drop below $30 \%$ of the nominal value in order to ensure survival of penicillin culture. Aeration is directly related to the DO level. To prevent DO level to drop below 30\%, a limiter is added to the valve so that it won't close more than a specific level.

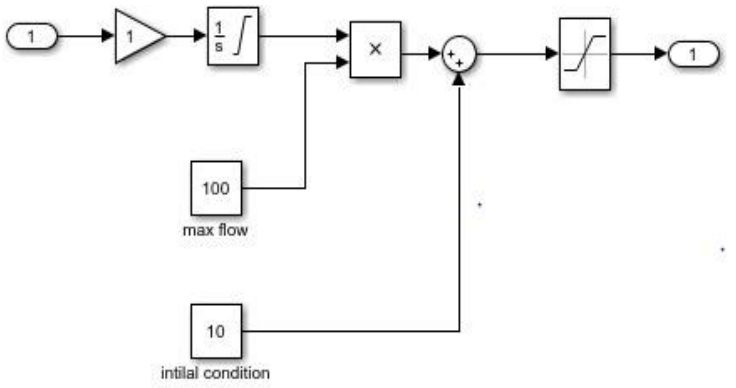

Figure 6. Valve subsystem in simulink

\subsection{Fuzzy Logic Controller (FLC)}

Before designing the fuzzy logic controller (FLC), the inputs to the fuzzy logic and its output should be determined. In this case, the output will change the opening of valve based current opening size as the opening of the valve will determine the aeration rate being feed into the bioreactor. Meanwhile, as the inputs, error of DO level of output to the set point and rate of change of error are taken. Figure 7 shows a simple representative of input and output of the fuzzy logic controller.

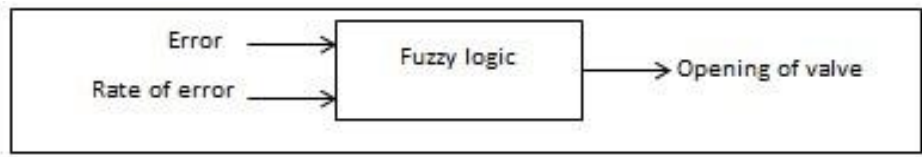

Figure 7. Structure of fuzzy logic with inputs and output 
The next step will be fuzzification which transforming the system inputs, which are crisp numbers, into fuzzy sets. For the input "error" it has three fuzzy sets which are "Low", "OK" and "High". Meanwhile, for the input "Rate of Error" it has three fuzzy sets as well: "Negative", "Zero", and "Positive". The output will have 5 distinct memberships: "Close Large", "Close Small", "No Change", "Open Small" and "Open large". Table 1 and Table 2 show the membership type of each input of "ERROR" and "RATE of ERROR" respectively including their crisp input range. Meanwhile Table 3 shows the membership function for the FLC's output. After fuzzification, a set of rules will be created. The rules are connected by "and" connectives. Table 4 shows the rule base of the FLC based on relationship of the inputs for the outputs.

To determine the output given input values, the FL should undergo defuzzification process. Defuzzification is the process of converting the degree of membership of output linguistic variable into crisp numerical values. For this study, centroid of gravity method is used.

Table 1. Membership of Each Variable of

\begin{tabular}{ccc}
\multicolumn{3}{c}{ Input "Error" } \\
\hline $\begin{array}{c}\text { Fuzzy } \\
\text { Variable }\end{array}$ & MF Used & $\begin{array}{c}\text { Crisp Input } \\
\text { Range }\end{array}$ \\
\hline High & Gaussian MF & $(3,-10)$ \\
OK & Gaussian MF & $(3,0)$ \\
Low & Gaussian MF & $(-3,10)$ \\
\hline
\end{tabular}

Table 3. Membership Function of Each Variable of Output "Valve Changes"

\begin{tabular}{ccc}
\hline $\begin{array}{c}\text { Fuzzy } \\
\text { Variable }\end{array}$ & MF Used & $\begin{array}{c}\text { Crisp Output } \\
\text { Range }\end{array}$ \\
\hline Close large & trimMF & $(0.8,1)$ \\
Close small & trimMF & $(0.2,0.4)$ \\
No change & trimMF & $(-0.1,0.1)$ \\
Open small & trimMF & $(-0.4,-0.2)$ \\
Open large & trimMF & $(-1,-0.8)$ \\
\hline
\end{tabular}

\section{RESULTS AND ANALYSIS}

\subsection{Sensitivity Analysis}

Sensitivity analysis was carried out on the dataset for batches 2, 3, 9 and 10 which are used for neural network training using cosine amplitude method to determine Rij that gives the relative significance of input variable's effect towards output variable. The higher value of Rij given between 0 and 1 indicates a more influential input variable. Figure 8 shows the result of sensitivity analysis for input variables; temperature (Celsius), water injection rate $(\mathrm{L} / \mathrm{m})$, sugar substrate feeding rate $(\mathrm{L} / \mathrm{m})$, pressure (bar) and aeration rate $(\mathrm{L} / \mathrm{m})$ on output variables; dissolved oxygen and penicillin concentration. From the results for two batches shown here, temperature and sugar substrate feeding rate has the biggest influence on dissolved oxygen and penicillin concentration respectively. The least significant variable for both output variables is water for injection. All other four input variables used in building the neural network models shows strong relationship to the output variable.

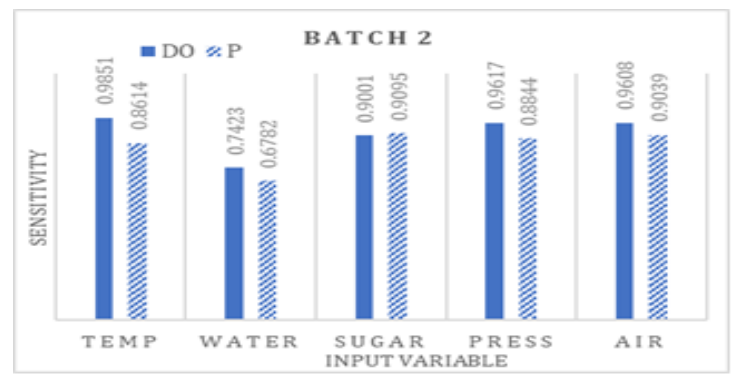

(a)

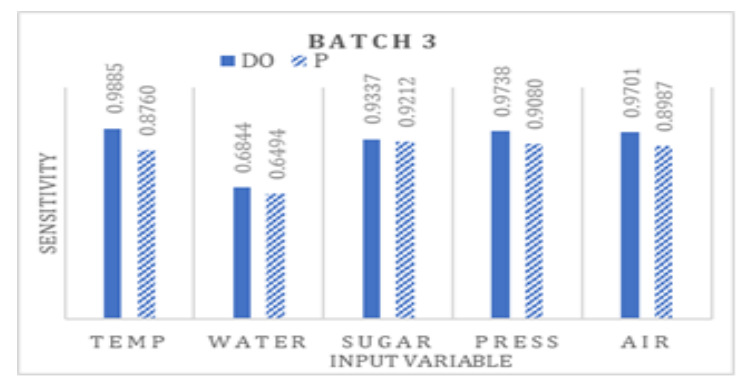

(b)

Figure 8. Sensitivity analysis for (a) batch 2 (b) batch 3 


\subsection{Block Diagram of the System in Simulink}

The project been simulated in Simulink MATLAB software using a block diagram that been designed in Simulink which consist of both model of the bioreactor and fuzzy logic controller to adjust the aeration rate to control the dissolved oxygen level in the bioreactor. The bioreactor plant consists of artificial neural network model for both DO level and penicillin concentration of the bioreactor. The negative feedback error of DO is fed into fuzzy logic controller as input. The negative feedback also been fed into differentiator block $(\mathrm{du} / \mathrm{dt})$ to get rate of change of error, which is the second input for the fuzzy logic. The opening size of valve is directly controlled by the fuzzy logic controller. The opening size of valve will determine the aeration rate $(\mathrm{L} / \mathrm{m})$ to be feed into the bioreactor model.

\subsection{Selection of ANN Model Structure}

Table 5 shows regression values of training and testing results for both MLP and RBF ANN obtained from regression plots. Regression analysis is a set of statistical processes for estimating the relationships among variables. In the case, relationship between the target output and trained output can be observed and analysed. A correlation coefficient or regression value R2 value closer to 1 indicates a closer fit of process output to model output. Table 5 compares the regression values to pick the best available trained neural network to be used as bioreactor model in simulation. For DO level, MLP shows higher regression value compared to RBF, which means it is better than RBF. However, the testing regression shows that RBF gives better fit compared to MLP. This shows that RBF perform better during the testing. This condition may occur due to MPL has been over-trained and becomes less flexible. Similar condition is seen in ANN training for penicillin concentration where MLP has higher regression value compare to RBF in training, but RBF has higher regression value in testing. This means that in both modelling, MLP has been over-trained, which made it less flexible compare to RBF ANN. Thus, RBF ANN is chosen as for both DO and penicillin concentration model to be used in simulation.

Table 5. Regression Values of Training and Testing Result for ANN

\begin{tabular}{cccc}
\hline Output Variable & ANN Method & $\begin{array}{c}\text { Training } \\
\text { Regression value }\end{array}$ & $\begin{array}{c}\text { Testing } \\
\text { Regression value }\end{array}$ \\
\hline D02 & MLP ANN & 0.9057 & 0.8630 \\
level & RBF ANN & 0.7981 & 0.8731 \\
Penicillin & MLP ANN & 0.9889 & 0.8860 \\
Concentration & RBF ANN & 0.9105 & 0.9105 \\
\hline
\end{tabular}

\subsection{Simulation Result}

Figure 9 shows DO level output of the bioreactor versus the DO set point that varies according time as defined by Profile 1 from batch 1 . The results show that the DO level successfully controlled to follow the profile with very small error by using fuzzy logic controller. The fuzzy logic controller also managed to eliminate overshoots occurrence in the response as normally seen in PID controller. The steady state error can be reduced by adjusting the error gain.

Figure 9 shows aeration rate as adjusted by the valve which controlled by the fuzzy logic controller. By manipulating aeration rate, DO level can be controlled. This relationship can be seen by comparing Figure 9 and Figure 10. As aeration rate is increased the DO level in the bioreactor also increases, this is true also for opposite condition.

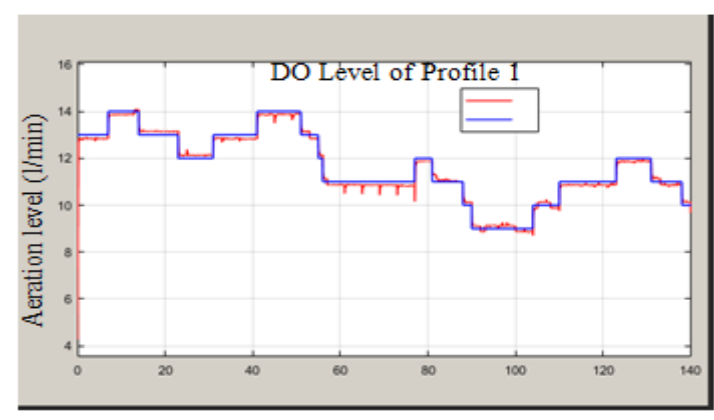

Figure 9. Simulated DO level using Profile 1

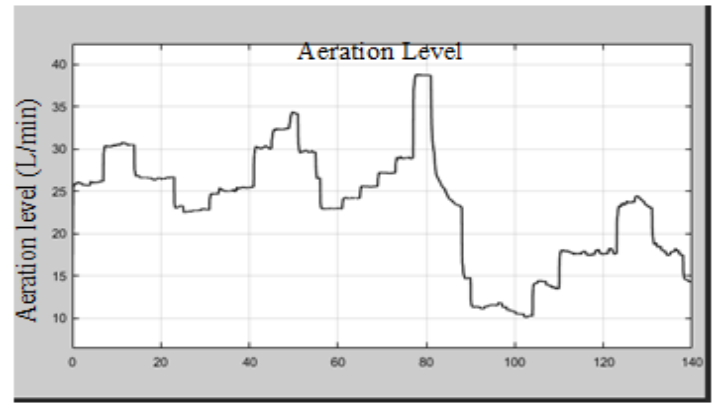

Figure 10. Simulated result of aeration rate to bioreactor controlled by Fuzzy Logic Controller for Profile 1 
Figure 11 shows that the penicillin concentration against time for Profile 1. The penicillin concentration increases as the fermentation duration increases as observed in the batch data. This shows that both model and controller successful in simulating the penicillin sp fermentation. The whole process took 140 hours. From the plot, it is observed that penicillin concentration reached its saturation at hour 90 . This means that, the process is completed by hour 100 and product can be collected by then. There is no need to wait until 140 hours to collect the product in the reactor. This is a fed batch process; hence whole product must be collected at the end of the process. The characteristic of the step response can be analysed based on Figure 12.

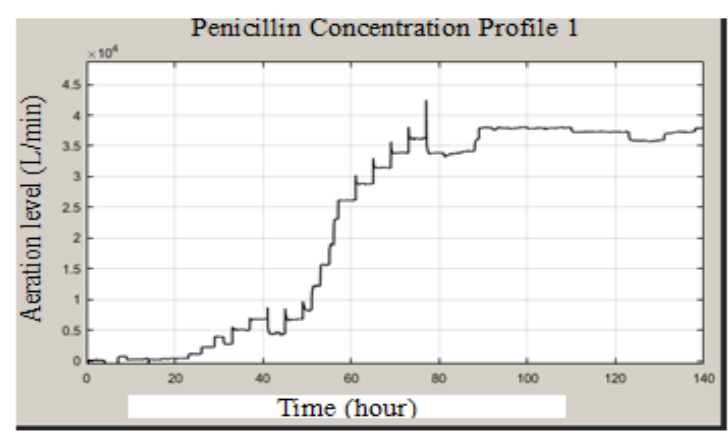

Figure 11. Simulated result of penicillin concentration in bioreactor culture for Profile 1

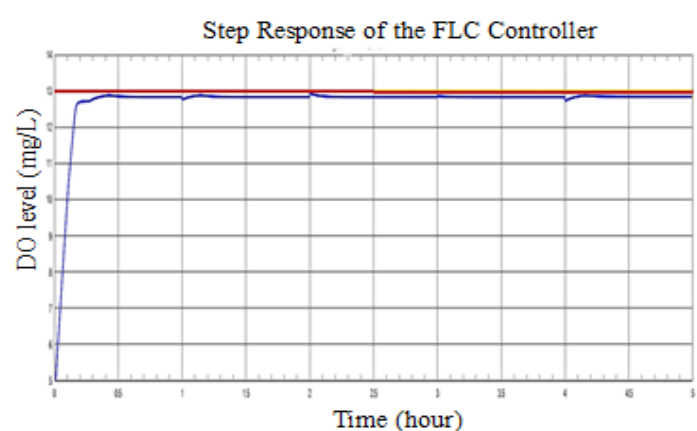

Figure 12: Step Response of the Fuzzy Logic Controller

A step response of $13 \mathrm{mg} / \mathrm{L}$ for DO level is given as the set point. Rise time, tr, it takes for the response to rise from $10 \%$ to $90 \%$ of the steady-state response. The rising time, tr, equivalent to 0.135 hours or 8.1 minutes. The step response of FLC shows that it does not have any overshoot. Steady-state error is defined as the difference between the set point and the output of a system in the limit as time goes to infinity. The output settles at $12.83 \mathrm{mg} / \mathrm{L}$ when the set-point is set to 13 . Therefore, it has steady state error of $0.17 \mathrm{mg} / \mathrm{L}$ or $1.307 \%$. This value is considered good as steady state error in bio-process is usually large. Settling time, ts is time it takes for the error between the response and set point to fall to within $2 \%$. In this case, the settling time is 0.2 hours or 12 minutes.

\section{CONCLUSION}

Both MLP and RBF neural network models show good results as shown by regression values for training and testing for DO2 level and penicillin concentration. Except for training regression value of RBF ANN for DO2 level around 0.8, all other regression values exceed 0.85. RBF neural network model shows better result as it shows no overfitting occurs. Simulation results for fuzzy logic controller shows that it can follow the reference profile for DO and penicillin concentration. The designed fuzzy logic controller can provide a good step response. Increasing the gain of error and rate of error in the circuit gives faster response, but there is chance to produce high noise level in the response, which is bad for the actuators such as the valve.

\section{ACKNOWLEDGEMENTS}

The authors would like to acknowledge the financial support of Universiti Putra Malaysia through Putra Grant Scheme with grant number 9552500.

\section{REFERENCES}

[1] A. Romo-Hernández, S. Hernández, A. Sánchez, and H. Hernández-Escoto, "A Comparative Study of MPC-Based Control Configurations of an Industrial Bioreactor to Produce Ethano"l, vol. 29. Elsevier B.V., 2011.

[2] M. C. Fernández, S. Rómoli, M. N. Pantano, O. A. O. D. Patiño, and G. J. E. Scaglia, "Controller design by monitoring desired concentration profiles for the penicillin production in a feed batch reactor," in XVI Workshop on Information Processing and Control (RPIC), 2015, pp. 1-6.

[3] M. Von Stosch, J. Hamelink, and R. Oliveira, "Hybrid modeling as a QbD / PAT tool in process development : an industrial E. coli case study," Bioprocess Biosyst. Eng., vol. 39, no. 5, pp. 773-784, 2016. 
[4] A. C. L. Horta, A. J. Da Silva, C. R. Sargo, V. M. Gonçalves, T. C. Zangirolami, and R. De Campos Giordano, "Robust artificial intelligence tool for automatic start-up of the supplementary medium feeding in recombinant E. coli cultivations," Bioprocess Biosyst. Eng., vol. 34, no. 7, pp. 891-901, 2011.

[5] Q. Jia et al., "Production of polyhydroxyalkanoates (PHA) by bacterial consortium from excess sludge fermentation liquid at laboratory and pilot scales," Bioresour. Technol., vol. 171, pp. 159-167, 2014.

[6] P. Biechele, C. Busse, D. Solle, T. Scheper, and K. Reardon, "Sensor systems for bioprocess monitoring," Eng. Life Sci., vol. 15, no. 5, pp. 469-488, 2015.

[7] X. Wang, A. Oehmen, E. B. Freitas, G. Carvalho, and M. A. M. Reis, "The link of feast-phase dissolved oxygen (DO) with substrate competition and microbial selection in PHA production," Water Res., vol. 112, pp. 269-278, 2017.

[8] G. Birol, C. Ündey, and A. Çinar, "A modular simulation package for fed-batch fermentation: Penicillin production," Comput. Chem. Eng., vol. 26, no. 11, pp. 1553-1565, 2002.

[9] A. Ashoori, B. Moshiri, A. Khaki-Sedigh, and M. R. Bakhtiari, "Optimal control of a nonlinear fed-batch fermentation process using model predictive approach,” J. Process Control, vol. 19, no. 7, pp. 1162-1173, 2009.

[10] H. Jin, X. Chen, J. Yang, L. Wu, and L. Wang, "Hybrid intelligent control of substrate feeding for industrial fedbatch chlortetracycline fermentation process," ISA Trans., vol. 53, no. 6, pp. 1822-1837, 2014.

[11] A. Pandey and J. A. C. Teixeira, Current Developments in Biotechnology and Bioengineering: Foundations of Biotechnology and Bioengineering. 2016.

[12] D. Bonne, M. A. Alvarez, and S. B. Jorgensen, "Data Driven Modeling for Monitoring and Control of Industrial Fed-Batch Cultivations," Ind. Eng. Chem. Res., vol. 53, no. 18, pp. 7365-7381, 2013.

[13] K. Hornik, M. Stinchcombe, and H. White, "Multilayer feedforward networks are universal approximators," Neural Networks, vol. 2, no. 5, pp. 359-366, 1989.

[14] M. Craninx, V. Fievez, B. Vlaeminck, and B. De Baets, "Artificial neural network models of the rumen fermentation pattern in dairy cattle," Comput. Electron. Agric., vol. 60, no. 2, pp. 226-238, 2008.

[15] L. Cui, P. Xie, J. Sun, T. Yu, and J. Yuan, "Data-driven prediction of the product formation in industrial 2-keto-1gulonic acid fermentation," Comput. Chem. Eng., vol. 36, no. 1, pp. 386-391, 2012.

[16] S. Velu, V. Velayutham, and S. Manickkam, "Optimization of fermentation media for xanthan gum production from Xanthomonas campestris using Response Surface Methodology and Artificial Neural Network techniques," Indian J. Chem. Technol., vol. 23, no. 5, pp. 353-361, 2016.

[17] S. Ö. Laursen, D. Webb, and W. F. Ramirez, "Dynamic hybrid neural network model of an industrial fed-batch fermentation process to produce foreign protein," Comput. Chem. Eng., vol. 31, no. 3, pp. 163-170, 2007.

[18] L. M. Rosales-Colunga, R. G. García, and A. De León Rodríguez, "Estimation of hydrogen production in genetically modified E. coli fermentations using an artificial neural network," Int. J. Hydrogen Energy, vol. 35, no. 24, pp. 13186-13192, 2010.

[19] Z. K. Nagy, "Model based control of a yeast fermentation bioreactor using optimally designed artificial neural networks," Chem. Eng. J., vol. 127, no. 1-3, pp. 95-109, 2007.

[20] A. C. Barchi et al., "Artificial intelligence approach based on near-infrared spectral data for monitoring of solidstate fermentation," Process Biochem., vol. 51, no. 10, pp. 1338-1347, 2016.

[21] L. Mears, S. M. Stocks, G. Sin, and K. V. Gernaey, "A review of control strategies for manipulating the feed rate in fed-batch fermentation processes," J. Biotechnol., vol. 245, pp. 34-46, 2017.

[22] S. Goldrick, A. Ştefan, D. Lovett, G. Montague, and B. Lennox, "The development of an industrial-scale fed-batch fermentation simulation," J. Biotechnol., vol. 193, pp. 70-82, 2015.

[23] S. Goldrick, B. Lennox, D. Lovett, K. Smith, and G. Montague, "The development of a simulation to address the real challenges associated with industrial scale penicillin production.," in IFAC Proceedings Volumes (IFACPapersOnline), 2013, vol. 12, no. PART 1, pp. 24-29.

[24] S. Ethaib, R. Omar, M. Kamal, S. Mazlina, A. Biak, and D. Radiah, "Development of a hybrid PSO - ANN model for estimating glucose and xylose yields for microwave-assisted pretreatment and the enzymatic hydrolysis of lignocellulosic biomass," Neural Comput. Appl., 2016.

[25] Y. Yang and Q. Zhang, "A hierarchical analysis for rock engineering using artificial neural networks," Rock Mech. Rock Eng., vol. 30, no. 4, pp. 207-222, 1997. 